Cite this: Phys. Chem. Chem. Phys., 2013

\title{
Mechanistic aspects of the linear stabilization of non-stationary electrochemical oscillations $\dagger$
}

15,1437

\author{
Murilo F. Cabral, ${ }^{a}$ Raphael Nagao, ${ }^{a}$ Elton Sitta, ${ }^{a}$ Markus Eiswirth ${ }^{\text {bc }}$ and \\ Hamilton Varela*ac
}

The problem of non-stationarity in experimentally recorded time-series is common in many (electro)chemical systems. Underlying this non-stationarity is the slow drift in some uncontrollable parameter, and it occurs in spite of the fact that all controllable parameters are kept constant. Particularly for electrochemical systems, some of us have recently suggested [J. Phys. Chem. C, 144, (2010), 22262-22268] an empirical method to stabilize experimental time-series. The method was exemplified for the electro-oxidation of methanol and different patterns were satisfactorily stabilized. In this paper we further elaborate some mechanistic aspects of this method and test it for the electrooxidation of formaldehyde, a system that has some resemblance with the electro-oxidation of methanol, but produces a richer dynamics. In terms of the reaction mechanism, we were able to describe the coupling and to separate the surface processes of the two sub-systems: the fast one (or the core-oscillator) and the slow one, responsible for the drift.

\begin{abstract}
Received 17th August 2012, Accepted 31st October 2012

DOI: $10.1039 / \mathrm{c} 2 \mathrm{cp} 42890 \mathrm{c}$
\end{abstract}

www.rsc.org/pccp
This spontaneous and uncontrollable drift has been found during the electro-oxidation of several organic molecules, ${ }^{9-12}$ and attributed to surface, rather than to transport processes. ${ }^{9-11,13}$ We coined the term uncompensated oscillations ${ }^{14}$ to describe the fact that the spontaneous drift slightly changes the surface composition, so that the population of different adsorbates at a given point in the phase-space does not remain constant after a single oscillatory cycle. In other words, after each cycle the surface population does not take on its original value before the cycle. As a consequence, a small accumulation and/or depletion of the surface population of the different adsorbates are/is observed. These changes are usually smaller than that observed in a single cycle and evolve slowly on top of the main oscillations. From the dynamic point-of-view, the whole system is characterized by the co-existence of two very different time-scales. The fast, main, dynamics is due to the core oscillator, whereas the secondary is due to the slow drift.

We have recently developed an empirical method, ${ }^{14}$ to stabilize the electrochemical potential oscillations in these systems. In the so-called negative galvanodynamic sweep (NGS), the applied current is linearly decreased in order to compensate the natural drift and thus stabilize the potential oscillations. The method was successfully applied for the oscillatory electrochemical oxidation of methanol on platinum, and distinct patterns were conveniently stabilized. In this paper, we further elaborate some mechanistic aspects of this method and test it for the electro-oxidation of formaldehyde. The electrocatalytic oxidation of formaldehyde ${ }^{15-18}$ has a much richer dynamics, ${ }^{7,9,19-22}$ 
but it still has some resemblances with that for methanol, despite being a by-product of the methanol electro-oxidation. ${ }^{15,23}$ In terms of the reaction mechanism, we were able to separate the surface processes of the coupled fast and slow sub-systems.

\section{Experimental}

The electrochemical experiments were carried out with a conventional electrochemical cell with three electrodes. The working electrode was a polycrystalline platinum foil with an area of $0.37 \mathrm{~cm}^{2}$ (electrochemical area $=0.48 \mathrm{~cm}^{2}$ ), a platinum net was employed as a counter electrode and the reference electrode was a reversible hydrogen electrode (RHE) prepared in the same acid solution of the electrolyte. The electrolyte solution was $0.5 \mathrm{M}$ $\mathrm{H}_{2} \mathrm{SO}_{4}$ (Mallinckrodt Baker) and the formaldehyde solution was prepared using formaldehyde solution (Mallinckrodt Baker). An amount of this solution was heated for $30 \mathrm{~min}$ and readily added in the electrochemical cell. The final concentration of formaldehyde in the electrochemical cell was equal to $0.1 \mathrm{M}$. Before each experiment the electrolyte solution was purged for at least $30 \mathrm{~min}$ with argon (AGA 99.99\%) and all solutions were prepared using ultrapure water from the Milli-Q Millipore ${ }^{\circledR}$ system. Before each experiment the working electrode was treated in propane/air flame and readily immersed in the electrolyte solution. The cleanness of the electrochemical apparatus was checked from successive cyclic voltammograms between 0.05 and $1.4 \mathrm{~V}$ at $0.1 \mathrm{~V} \mathrm{~s}^{-1}$ until the characteristic voltammetric profile for the polycrystalline platinum electrode in acid media was observed. Electrochemical cell potentials were controlled with a potentiostat/galvanostat (Autolab/Eco-Chemie, PGSTAT3002) equipped with SCANGEN module. Oscillatory patterns were also visually monitored by means of a Minipa oscilloscope (model MO-1225 $20 \mathrm{MHz}$ ). All experiments were carried out at $25{ }^{\circ} \mathrm{C}$.

\section{Results and discussion}

Fig. 1 shows the cyclic voltammetry of the platinum electrode in both (a) the base electrolyte, and (b) after addition of $0.1 \mathrm{M}$ HCHO. The overall features of the voltammetric profile for the electro-oxidation of formaldehyde under similar conditions, as well their relationship with the base cyclic voltammogram, can be found elsewhere. ${ }^{9,24}$

The potential response to a galvanodynamic sweep registered at $10.42 \mu \mathrm{A} \mathrm{cm} \mathrm{cm}^{-2} \mathrm{~s}^{-1}$ is also shown in Fig. 1(b). Potential oscillations develop at a considerably low applied current (and $U \sim 0.60 \mathrm{~V}$ ) and persist over the whole intermediate values. The dynamics of the electro-oxidation of formaldehyde under potential and current control can be compared to that of methanol, ${ }^{14}$ for instance. The main difference to be stressed is the fact that the amplitudes observed here are considerably larger than that for methanol. Indeed, potential oscillations in the present case cover the potential range of about $0.75 \mathrm{~V}$ whereas for methanol it remains around $0.20 \mathrm{~V}^{14}$ The large amplitudes found for the potential oscillations in the case of formaldehyde reflect mainly the excursions into the low potential region, as further discussed below.

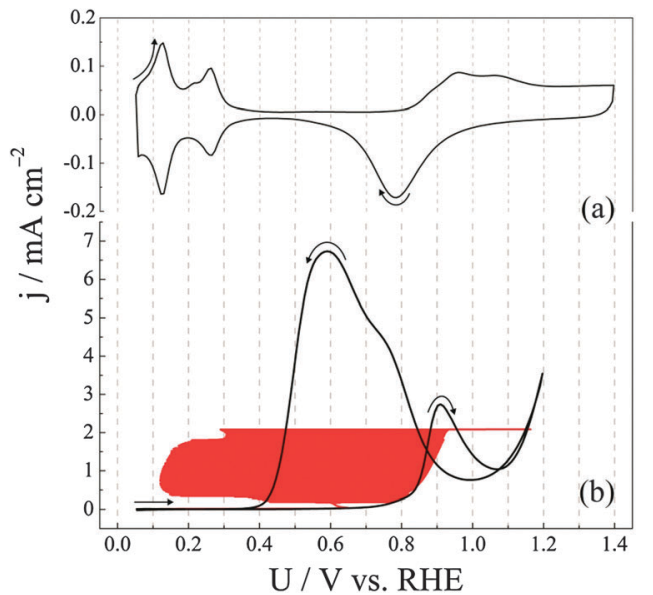

Fig. 1 Cyclic voltammograms (black) for the platinum electrode in (a) $0.5 \mathrm{M}$ $\mathrm{H}_{2} \mathrm{SO}_{4}$ aqueous solution, and (b) after addition of $0.1 \mathrm{~mol} \mathrm{~L}^{-1}$ of $\mathrm{HCHO}$. Galvanodynamic sweep (red) obtained at $10.42 \mu \mathrm{A} \mathrm{cm} \mathrm{cm}^{-2} \mathrm{~s}^{-1}$. Both cyclic voltammograms were recorded at $0.1 \mathrm{~V} \mathrm{~s}^{-1}$.

A detailed view of the galvanodynamic sweep shown in Fig. 1(b) is presented in Fig. 2(b), along with the sequence of six distinct temporal patterns in panels (c)-(h). In (a) the evolution of the mean potential, $U_{\mathrm{m}}$, estimated for each cycle between $t_{1}$ and $t_{2}$ is shown,

$$
U_{\mathrm{m}}=\frac{\int_{t_{1}}^{t_{2}} U(t) \mathrm{d} t}{t_{2}-t_{1}}
$$

The sequence of six selected windows illustrates the continuous transitions among different oscillatory patterns observed as the current is slowly increased. This sequence is, at least partially, captured at different sweep rates. Overall, most structures were already reported by Okamoto and co-workers. ${ }^{9-11,19,24-27}$ The first pattern, panel (c), is characterized by a slow potential increase followed by a fast one and a subsequent decrease. These oscillations repeat for seven cycles and are the ones of lowest amplitude. The large amplitude oscillations shown in (e) are the most stable ones observed during the galvanodynamic sweep (240 cycles), and the fast potential decrease reaches the lowest values during the series. The transition between the series presented in (c) and (e) is mediated by composite patterns (d). These complex structures are rather unstable and exist for only two cycles, see also Fig. 4 in ref. 27. The pattern in (f) is similar to the previous one but has a small modulation in the high potential region. The main feature of the next structures, $(\mathrm{g})$ and $(\mathrm{h})$, is the concomitant occurrence of the slow potential increase (in a similar potential region as that of pattern (c)) and longer excursion into a high potential region, as in patterns (e) and (f). When compared to the oscillations presented in (c), the longer stay in the high potential region results in a more effective oxidation of adsorbed species and, consequently, a following deeper potential decrease. This observation is in line with our previous report on the electro-oxidation of $\mathrm{H}_{2}-\mathrm{CO}$ mixtures in a PEM fuel cell. ${ }^{28}$ The patterns presented in Fig. $2(\mathrm{~g})$ and (h) appear for 37 and 7 cycles, respectively, and clearly belong to the same family, the only slight difference between them is the format of time spent in the high potential region. 

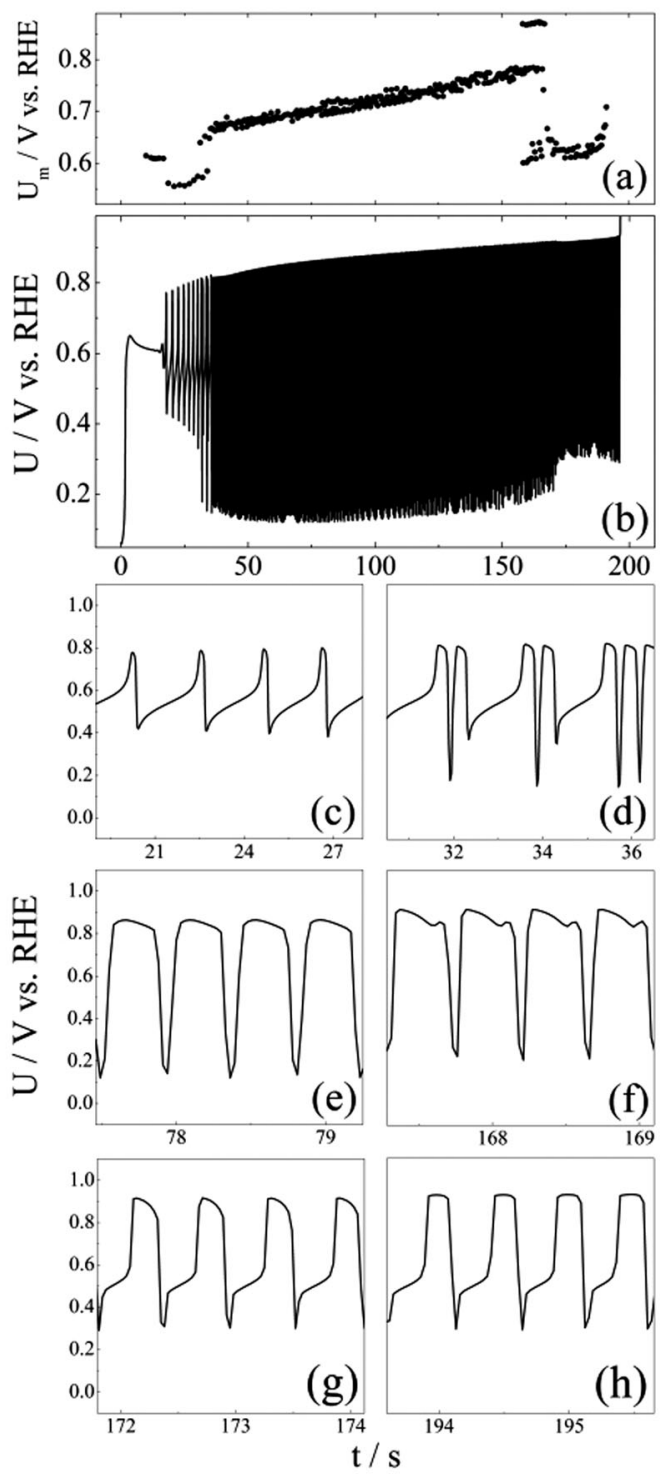

Fig. 2 Time-traces of (a) the mean electrode potential, and (b) the electrode potential, during the galvanodynamic electro-oxidation of formaldehyde on platinum. Details of the oscillations are depicted in selected windows in (c)-(h). The experimental conditions are the same as in Fig. 1(b).

A qualitative analysis of the oscillation amplitude can provide valuable information. Large excursions into the low potential regions is common for formaldehyde and also formic acid, ${ }^{20,25,29-31}$ but are apparently not observed in the oscillatory electro-oxidation of methanol. ${ }^{7-9,14,22,24,32,33}$ The potential oscillations reflect the changes in the population of different adsorbed intermediates. In short, the process can be phenomenologically described as follows. When the population of poisoning species (mainly adsorbed carbon monoxide in the case of $\mathrm{C} 1$ molecules, vide infra) increases, the number of available sites decreases so that the electrode potential increases in order to allow the oxidation of poisoning species and thus to accomplish the applied current. When the strongly adsorbed poisoning species are oxidized, the potential decreases and the cycle starts anew. Therefore, one can expect that the amplitude of the potential oscillations is correlated with the amplitude of the oscillating coverages of some adsorbed species. This conjecture has been found for the oscillating coverage of adsorbed carbon monoxide for methanol and formic acid. ${ }^{7}$ In terms of absolute values, the lower potential values observed for formaldehyde indicates that the direct pathway is more active (vide infra) than that for methanol. These are apparently the cause of simpler oscillatory dynamics found for methanol when compared to that for formic acid and formaldehyde. ${ }^{7}$ This aspect, and thus the variety of the oscillatory patterns, justifies the choice of the electro-oxidation of formaldehyde to further test our stabilization method. ${ }^{14}$

The continuous transitions among different structures can be discussed in terms of the evolution of the mean electrode potential, $c f$. Fig. 2(a). There are clearly three distinct oscillatory regions. The first region consists of considerably low potential values and it is associated to the first oscillations shown in Fig. 2(c). As already said, most of the oscillations in this series consist of the patterns shown in parts (e) and (f) and along this region the mean electrode potential increases consistently at a rate of $1.76 \mathrm{mV} \mathrm{s}^{-1}$. In the last potential region, the mean electrode potential drops again but takes on slightly larger values, as already mentioned in the time spent in the high potential region of the series in $(\mathrm{g})$ and $(\mathrm{h})$ when compared to that in (c). Along the galvanodynamic sweep the potential increase in this last window results in the explosive-like increase of the electrode potential and thus the extinction of the oscillations. Under the galvanostatic regime, however, it is possible to observe the development of high amplitude oscillations after the ones presented in parts $(\mathrm{g})$ and $(\mathrm{h})$. The abrupt changes in the mean electrode potential observed in Fig. 2(a) are fairly in contrast to that found for methanol, ${ }^{14}$ where an inexorable increase of the mean electrode potential prevails. The formaldehyde system can thus be considered more robust than the methanol one in the sense that oscillations can exist in a wider parameter range. This observation is corroborated by the richer dynamics observed in the electro-oxidation of formaldehyde.

Truly galvanostatic experiments can be used to investigate individual oscillatory states, the problem, however, in such experiments is that the system does not remain strictly stationary due to the slow variation in some uncontrollable parameter. As a consequence, a slow transition among oscillatory states is observed, following the general trend observed when the current is varied, $c f$. Fig. 2. However, in such galvanostatic experiments, the first pattern to appear and the completeness of the sequence depends on the applied current. We applied the negative galvanodynamic sweep (NGS $)^{14}$ to some of the patterns shown in Fig. 2, but systematically studied two of them, namely the ones shown in Fig. 2(f) and (g)/ (h). These patterns were selected because of the difference in the mean potential around which oscillations develop.

Fig. 3 shows the number of cycles as a function of the applied NGS, the reference value is given for the galvanostatic case, i.e. for $\mathrm{d} j / \mathrm{d} t=0$. The initial applied current was $1.7 \mathrm{~mA} \mathrm{~cm}{ }^{-2}$ and, under the strictly galvanostatic regime, 37 and 48 cycles were observed for patterns shown in Fig. 2(f) and (g), respectively. The negative galvanodynamic sweep was started at $1.7 \mathrm{~mA} \mathrm{~cm} \mathrm{~cm}^{-2}$ 


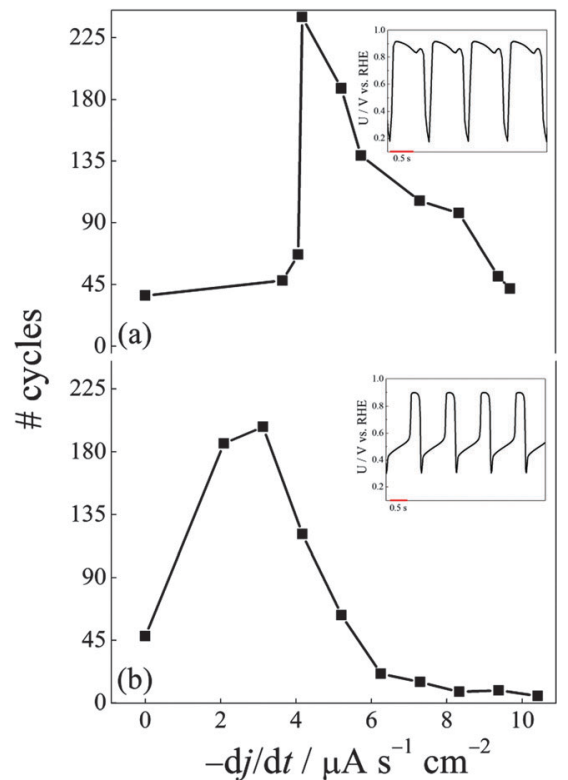

Fig. 3 Effect of the applied negative galvanodynamic sweep on the number of oscillatory cycles for two selected temporal patterns.

when the target pattern appears. A well-defined maximum in the number of cycles was obtained for each pattern and amounts to a stabilization of 6.5 (or 240 cycles at $-4.17 \mu \mathrm{A} \mathrm{s}^{-1} \mathrm{~cm}^{-2}$ ) times for the oscillations shown in Fig. 2(f) and to a 4 times (198 cycles for $\mathrm{d} j / \mathrm{d} t=-3.13 \mu \mathrm{A} \mathrm{s}^{-1} \mathrm{~cm}^{-2}$ ) stabilization for that presented in Fig. $2(\mathrm{~g}) /(\mathrm{h})$. The reason for the occurrence of a maximum at a given sweep rate was discussed in our previous report, ${ }^{14}$ which indicates the optimum rate of current decrease that, at least partially, compensates the spontaneous surface deactivation.

Fig. 4 illustrates the effect of the NGS on the stabilization of the pattern shown in Fig. 3(b). Results are presented for

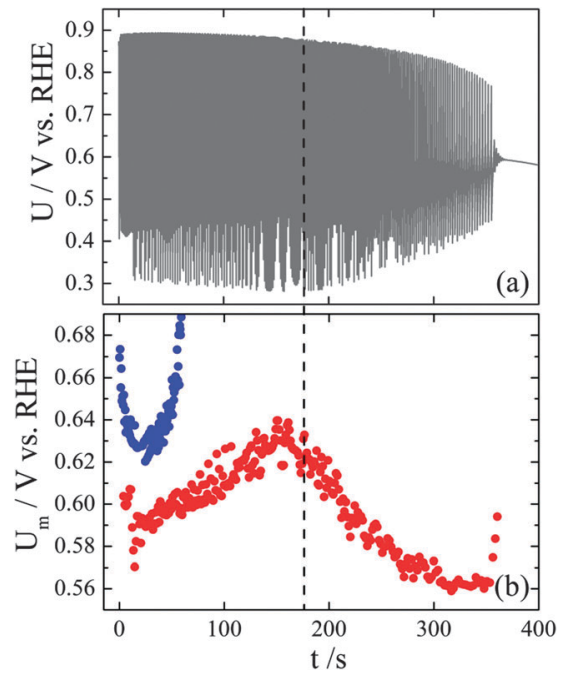

Fig. 4 (a) Stabilized time-series presented in Fig. 3(b); (b) evolution of the mean electrode potential for the native time-series (blue) and for the stabilized case (red). The vertical dashed line delimitates the region $(0<t<\sim 180 \mathrm{~s})$ where the stabilized pattern is found. $\mathrm{d} j / \mathrm{d} t=-3.13 \mu \mathrm{A} \mathrm{s}^{-1} \mathrm{~cm}^{-2}$, which corresponds to the stabilization of 198 cycles. The data in (a) account for the whole experiment and the stabilization window consists of the appearance of the desired oscillatory pattern and also to the point where the NGS was launched (at $t=0)$ up to the time ( $c a .180 \mathrm{~s}$ ) where this pattern remains stable. From this point on, a smaller amplitude structure equivalent to the one shown in Fig. 2(c) prevails and eventually the current becomes so small that oscillations extinguish. The apparent irregularity in the timeseries as a whole comes from the limitation of our data-acquisition system, but both the identification of a given pattern and the quantification of the number of cycles were assured by a careful parallel on-time inspection with an oscilloscope.

Fig. 4(b) shows the dynamics of $U_{\mathrm{m}}$ for the stabilized timeseries shown in (a) and also for the same pattern without any NGS (in blue), i.e. for the situation presented in Fig. 3(b) when $\mathrm{d} j / \mathrm{d} t=0$. In spite of the scattering in the absolute values of the mean electrode potential, it is clear in this plot that the application of the NGS slows down the natural drift and thus increases the number of cycles. After about $180 \mathrm{~s}$, the target pattern becomes unstable and $U_{\mathrm{m}}$ decreases as a different structure develops. After some time the applied current becomes so small that oscillations fade away.

Despite the differences in the mechanistic aspects associated to the oscillatory amplitude, already discussed, there are also important similarities between the electro-oxidation of formaldehyde and methanol. With respect to the dynamic instabilities, both systems belong to the class of HN-NDR electrochemical oscillators, ${ }^{34-36}$ and, as observed in the case of methanol, ${ }^{7,14}$ the secondary slow process associated to the spontaneous drift is also reflected in the increase of the mean electrode potential.

Bringing together the results for the stabilization of time-series for formaldehyde and also for methanol, ${ }^{14}$ we can now suggest a general mechanism for the instabilities associated to both the core oscillator and the slow drift. Fig. 5 sketches the main steps of a generalized mechanism encompassing the dual pathway that leads to carbon dioxide in the electro-oxidation of many small organic molecules. ${ }^{37-39}$ The scheme is very general and it is aimed to represent the electro-oxidation of formaldehyde, and also of methanol, on platinum. For formaldehyde, the initial species A accounts for bulk methylene glycol (the predominant form of formaldehyde in aqueous acidic media ${ }^{16,40-43}$ ) and species B is the adsorbed form which occupies $x$ platinum sites. In the case of methanol, A is a solution methanol molecule, and B represents an adsorbed HCO species, which in turn is formed after the adsorptive dehydrogenation of a $\mathrm{H}_{3} \mathrm{COH}$ molecule - a process that requires three contiguous sites. ${ }^{44}$ Species HCO is believed to be adsorbed either via the carbon or the oxygen

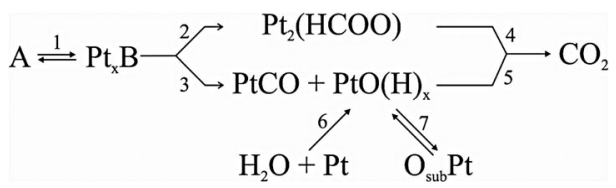

Fig. 5 Reaction scheme of the electro-oxidation of methanol and formaldehyde on platinum. See the text for details. 
atom, and this configuration will determine if this adsorbate will follow the direct or the indirect pathway. ${ }^{23,45,46}$

Starting from the generic $\mathrm{Pt}_{x} \mathrm{~B}$ species, there are two parallel pathways that result in the formation of carbon dioxide. In short, the direct pathway, steps 2 and 4, occurs via the formation of an active intermediate, presumably, adsorbed formate. ${ }^{4,19,47-49}$ The formation of formate from the generic precursor $\mathrm{Pt}_{x} \mathrm{~B}$ is still somewhat under debate for both formaldehyde and methanol, mainly because of the need of an additional oxygenated species. Nevertheless, spectroscopic experiments give evidences of the presence of adsorbed formate at a lower potential than that expected for the water discharge, step 6. Adsorbed formate might also result in adsorbed $\mathrm{CO}$ or in dissolved $\mathrm{HCOOH}$, those possibilities were omitted in Fig. 5 for the sake of simplicity. The indirect pathway proceeds via formation of adsorbed CO, which remains blocking surface sites and gets oxidized at considerably high potentials where oxygenated species are formed at the electrode surface.

In terms of oscillatory dynamics, steps 1 to 6 in Fig. 5 are assumed to be part of the core oscillator, and specificities due to the use of formaldehyde or methanol are mainly connected to the site requirement of particular steps, rate constants of individual steps, relative surface populations, etc. The surface species $\mathrm{PtO}(\mathrm{H})_{x}$ accounts for a generic oxygenated species that helps the electro-oxidation of species such as adsorbed carbon monoxide. The increase in the population of such species results in the process of place-exchange, ${ }^{50}$ in which surface oxygen atoms are inserted in the platinum lattice in order to allow further surface oxidation and thus accommodate more oxygenated species. In terms of surface reactions, sub-surface oxygen would not be available for Langmuir-Hinshelwood steps such as the illustrated electro-oxidation of adsorbed carbon monoxide. In addition, once both oxygen and platinum atoms in the $\mathrm{O}_{\text {sub }}-\mathrm{Pt}$ species are unavailable surface sites, the increase in the amount of $\mathrm{O}_{\mathrm{sub}}-\mathrm{Pt}$ accompanying the increase in the electrode potential is equivalent to a decrease in the overall number of surface sites. As a consequence, this decrease causes an increase in the actual current density (in contrast to the static, applied current, which is strictly constant). Finally, as the increase of $U_{\mathrm{m}}$ reflects the increase of surface oxidation and thus the amount of sub-surface species, we suggest the formation of sub-surface oxygen as the main cause for the uncompensated oscillations.

As simplified in step 7, this is a reversible process in the sense that sub-surface oxygen, $\mathrm{O}_{\text {sub }}-\mathrm{Pt}$, might also emerge to the surface and thus help in the oxidation of a carbon residue. As presented in Fig. 2(a), the consumption of oxygenated species, and the emergence of sub-surface oxygen, seems to be reflected in the pronounced decrease in $U_{\mathrm{m}}$ after its slow increase along the drift. In the case of methanol, the inexorable increase in $U_{\mathrm{m}}$ is likely to be due to the narrower potential window where oscillations develop.

\section{Conclusions}

The use of a negative galvanodynamic sweep was successfully applied to the stabilization of non-stationary potential oscillations during the electro-oxidation of formaldehyde on polycrystalline platinum surfaces. In the parameter region studied, five temporal patterns were observed, and two were chosen and conveniently stabilized. These results extend our previous findings for methanol, ${ }^{14}$ a system that is mechanistically comparable to the formaldehyde one, but posseses a much simpler dynamics. In addition to the mere extension, however, we furtherer our understanding of the dynamics associated to the non-stationarity observed in experimentally recorded electrochemical time-series, a common occurrence in many systems. The reaction mechanism was discussed in terms of the surface processes underlying the dynamics of the slow and fast subsystems. The fast dynamics, or the oscillations in the core system, is associated to the coverage changes in the populations of species such as adsorbed carbon monoxide, oxygenated species, adsorbed formate, and so on. The slow drift, reflected in the gradual increase of $U_{\mathrm{m}}$, was suggested to be caused by the increase of surface oxidation and thus the amount of sub-surface oxygen, formed by the place exchange process. In other words, the slow and uncontrollable drift is due to the transformation of surface to sub-surface oxygenated species. Sub-surface oxygen species are not available to oxidize adsorbed carbonaceous poisoning species, and therefore are not expected to take part in the oscillatory cycle.

\section{Acknowledgements}

The authors acknowledge Ms B. C. Batista for fruitful discussions, and the following agencies for funding: Conselho Nacional de Desenvolvimento Científico e Tecnológico (CNPq, Grants No. 141455/2008-0 (ES), 306151/2010-3 (HV), and 450286/2012-6 (HV)), and Fundação de Amparo à Pesquisa do Estado de São Paulo (FAPESP, Grants No. 09/07629-6 (HV), and 2012/07882-6 (MFC), 2009/00153-6 (RN)) for financial support.

\section{References}

1 M. Hachkar, B. Beden and C. Lamy, J. Electroanal. Chem., 1990, 287, 81-98.

2 K. Krischer and H. Varela, in Handbook of Fuel Cells: Fundamentals, Technology, Applications., ed. W. Vielstich, H. A. Gasteiger and A. Lamm, John Wiley \& Sons, Chichester, 2003, vol. 2, p. 679.

3 U. Krewer, T. Vidakovic-Koch and L. Rihko-Struckmann, ChemPhysChem, 2011, 12, 2518-2544.

4 G. Samjeské, A. Miki, S. Ye, A. Yamakata, Y. Mukouyama, H. Okamoto and M. Osawa, J. Phys. Chem. B, 2005, 109, 23509-23516.

5 G. Samjeské and M. Osawa, Angew. Chem., Int. Ed., 2005, 44, 5694-5698.

6 G. Samjeské, A. Miki and M. Osawa, J. Phys. Chem. C, 2007, 111, 15074-15083.

7 E. Boscheto, B. C. Batista, R. B. Lima and H. Varela, J. Electroanal. Chem., 2010, 642, 17-21.

8 R. Nagao, D. A. Cantane, F. H. B. Lima and H. Varela, Phys. Chem. Chem. Phys., 2012, 14, 8294-8298. 
9 H. Okamoto, N. Tanaka and M. Naito, J. Phys. Chem. A, 1997, 101, 8480-8488.

10 H. Okamoto, N. Tanaka and M. Naito, J. Phys. Chem. A, 1998, 102, 7353-7361.

11 H. Okamoto, N. Tanaka and M. Naito, J. Phys. Chem. A, 1998, 102, 7343-7352.

12 E. Sitta, M. A. Nascimento and H. Varela, Phys. Chem. Chem. Phys., 2010, 12, 15195-15206.

13 H. Okamoto, M. Kikuchi and Y. Mukouyama, J. Electroanal. Chem., 2008, 622, 1-9.

14 R. Nagao, E. Sitta and H. Varela, J. Phys. Chem. C, 2010, 114, 22262-22268.

15 E. A. Batista, H. Hoster and T. Iwasita, J. Electroanal. Chem., 2003, 554-555, 265-271.

16 E. A. Batista and T. Iwasita, Langmuir, 2006, 22, 7912-7916.

17 R. B. de Lima, M. P. Massafera, E. A. Batista and T. Iwasita, J. Electroanal. Chem., 2007, 603, 142-148.

18 N. Markovic and P. N. Ross, J. Phys. Chem., 1993, 97, 9771-9778.

19 M. Kikuchi, W. Kon, S. Miyahara, Y. Mukouyama and H. Okamoto, Electrochim. Acta, 2007, 53, 846-852.

20 F. N. Albahadily and M. Schell, J. Electroanal. Chem., 1991, 308, 151-173.

21 M. Schell and X. Cai, Electrochim. Acta, 1993, 38, 519-527.

22 A. L. Martins, B. C. Batista, E. Sitta and H. Varela, J. Braz. Chem. Soc., 2008, 19, 679-687.

23 E. A. Batista, G. R. P. Malpass, A. J. Motheo and T. Iwasita, J. Electroanal. Chem., 2004, 571, 273-282.

24 H. Okamoto, W. Kon and Y. Mukouyama, J. Phys. Chem. B, 2005, 109, 15659-15666.

25 H. Okamoto and N. Tanaka, Electrochim. Acta, 1993, 38, 503-509.

26 H. Okamoto, N. Tanaka and M. Naito, Electrochim. Acta, 1994, 39, 2471-2474.

27 H. Okamoto, N. Tanaka and M. Naito, J. Electrochem. Soc., 2000, 147, 2629-2635.

28 A. Mota, P. P. Lopes, E. A. Ticianelli, E. R. Gonzalez and H. Varela, J. Electrochem. Soc., 2010, 157, B1301-B1304.

29 M. Schell, F. N. Albahadily, J. Safar and Y. Xu, J. Phys. Chem., 1989, 93, 4806-4810.

30 J. Lee, J. Christoph, P. Strasser, M. Eiswirth and G. Ertl, Phys. Chem. Chem. Phys., 2003, 5, 935-938.
31 R. Nagao, I. R. Epstein, E. R. Gonzalez and H. Varela, J. Phys. Chem. A, 2008, 112, 4617-4624.

32 M. Krausa and W. Vielstich, J. Electroanal. Chem., 1995, 399, 7-12.

33 E. A. Carbonio, R. Nagao, E. R. Gonzalez and H. Varela, Phys. Chem. Chem. Phys., 2009, 11, 665-670.

34 K. Krischer, in Modern Aspects of Electrochemistry: Principles of Temporal and Spatial Pattern Formation in Electrochemical Systems, ed. B. E. Conway, J. O. M. Bockris and R. White, Springer US, 1999, vol. 32, ch. 1, pp. 1-142.

35 P. Strasser, M. Eiswirth and M. T. M. Koper, J. Electroanal. Chem., 1999, 478, 50-66.

36 M. T. M. Koper, in Adv. Chem. Phys., ed. I. Prigogine and S. A. Rice, John Wiley \& Sons, Hoboken, 1996, vol. 92, p. 161.

37 G. A. Attard, H. D. Ebert and R. Parsons, Surf. Sci., 1990, 240, 125-135.

38 R. Parsons, J. Electrochem. Soc., 1980, 127, 176C-182C.

39 R. Parsons and T. VanderNoot, J. Electroanal. Chem., 1988, 257, 9-45.

40 Y. Kunugi, Y. Ono and T. Nonaka, J. Electroanal. Chem., 1992, 333, 325-329.

41 P. Olivi, L. O. S. Bulhões, J. M. Léger, F. Hahn, B. Beden and C. Lamy, J. Electroanal. Chem., 1994, 370, 241-249.

42 P. Olivi, L. O. S. Bulhões, J. M. Léger, F. Hahn, B. Beden and C. Lamy, Electrochim. Acta, 1996, 41, 927-932.

43 S. Nakabayashi, I. Yagi, N. Sugiyama, K. Tamura and K. Uosaki, Surf. Sci., 1997, 386, 82-88.

44 A. Cuesta, J. Am. Chem. Soc., 2006, 128, 13332-13333.

45 T. H. M. Housmans, A. H. Wonders and M. T. M. Koper, J. Phys. Chem. B, 2006, 110, 10021-10031.

46 S. Lai, N. Lebedeva, T. Housmans and M. Koper, Top. Catal., 2007, 46, 320-333.

47 Y. X. Chen, A. Miki, S. Ye, H. Sakai and M. Osawa, J. Am. Chem. Soc., 2003, 125, 3680-3681.

48 Y. Mukouyama, M. Kikuchi, G. Samjeské, M. Osawa and H. Okamoto, J. Phys. Chem. B, 2006, 110, 11912-11917.

49 M. Osawa, K. Komatsu, G. Samjeské, T. Uchida, T. Ikeshoji, A. Cuesta and C. Gutiérrez, Angew. Chem., Int. Ed., 2011, 50, 1159-1163.

50 H. Angerstein-Kozlowska, B. E. Conway and W. B. A. Sharp, J. Electroanal. Chem., 1973, 43, 9-36. 